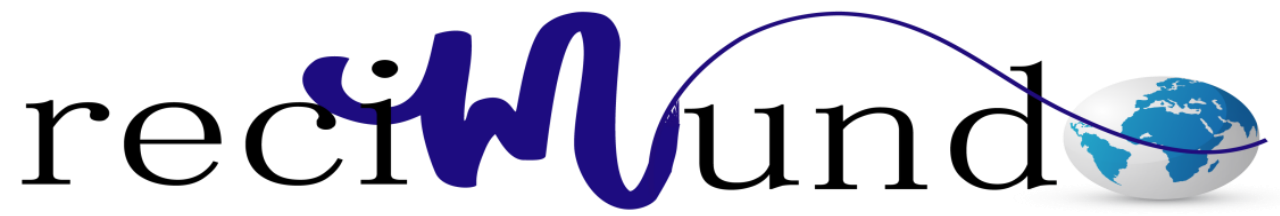

Revista Cientifica Mundo de la Investigación y el Conocimiento

Gladys Patricia Guevara Albán ${ }^{\text {a }}$; Cristian Salomón Guevara Albán ${ }^{\text {b; }}$ Alexis Eduardo Verdesoto Arguello ${ }^{c}$

Redes Informáticas aplicadas a la Educación

Revista Científica Mundo de la Investigación y el Conocimiento. Vol. 2 núm.2, mayo, ISSN: 2588-073X, 2018, pp. 24-44

DOI: 10.26820/recimundo/2.(2).2018.24-44

Editorial Saberes del Conocimiento

Recibido: 05/12/2017

Aceptado: 17/03/2018
a. Universidad Técnica de Babahoyo; gguevara@utb.edu.ec
b. crisalo1991@gmail.com
c. Instituto Tecnológico Superior Babahoyo; averdesoto@itsb.edu.ec 


\section{Redes Informáticas aplicadas a la Educación}

Vol. 2, núm. 2., (2018)

Gladys Patricia Guevara Albán; Cristian Salomón Guevara Albán; Alexis Eduardo Verdesoto

Arguello

\section{RESUMEN}

El presente proyecto de investigación se basa en la descripción, definición de términos y conceptualización en líneas generales de lo concerniente al uso de las tecnologías de la información y comunicación (TIC's) en la comunidad educativa, haciendo énfasis en la utilización de las redes informáticas en las aulas de clase, vistas como una herramienta pedagógica hoy en día indispensable para el fortalecimiento de las actividades curriculares. Constantemente es necesario implementar el desarrollo formativo, pedagógico y didáctico que contribuya con el país principalmente en los cambios sociales, culturales, económicos y políticos, por ende, la utilización de las TIC's en la aplicación del proceso de interaprendizaje educativo como parte de una formación integral es imprescindible, de conformidad al contexto sociocultural que se está viviendo a nivel mundial. Por esta razón es menester promover una formación técnica de docentes mediante los materiales esenciales para la aplicación de nuevos modelos educativos a favor de los alumnos con la implementación de los equipos tecnológicos necesarios. La metodología empleada en la presente investigación se hizo en base a un enfoque documental, haciendo uso de una revisión bibliográfica a nivel exploratorio-descriptivo, mediante una laboriosa búsqueda de información, tanto en publicaciones hechas en material físico como las hechas por medios electrónicos, tales como libros, revistas especializadas, foros, simposios, páginas web, e-book y demás fuentes de información, nacionales e internacionales, legales, oficiales o extraoficiales, respaldadas por profesionales en la materia, en idioma castellano, y en todo momento relacionadas con el tema en cuestión, todo ello con la primordial intención de que tal información sirva para proporcionar conocimiento fundamental al interesado, y en particular para esta investigación, de fundamento de las conclusiones que se desarrollarán a continuación, y aporte formativo para posibles investigaciones del tema.

Palabras clave: Tecnologías, información, comunicación, redes, modelo. 


\title{
Redes Informáticas aplicadas a la Educación
}

Vol. 2, núm. 2., (2018)

Gladys Patricia Guevara Albán; Cristian Salomón Guevara Albán; Alexis Eduardo Verdesoto Arguello

\begin{abstract}
This research project is based on the description, definition of terms and conceptualization in general terms of what concerns the use of information and communication technologies (ICTs) in the educational community, emphasizing the use of computer networks in classrooms, seen as a pedagogical tool nowadays indispensable for the strengthening of curricular activities. It is constantly necessary to implement the educational, pedagogical and didactic development that contributes with the country mainly in the social, cultural, economic and political changes, therefore, the use of the TIC's in the application of the educational inter-learning process as part of an integral formation it is essential, in accordance with the socio-cultural context that is being experienced worldwide. For this reason it is necessary to promote technical training for teachers through the essential materials for the application of new educational models in favor of students with the implementation of the necessary technological equipment. The methodology used in the present investigation was based on a documentary approach, making use of a bibliographic review at the exploratory-descriptive level, through a laborious search of information, both in publications made in physical material and those made by electronic means, such such as books, specialized magazines, forums, symposia, web pages, e-books and other sources of information, national and international, legal, official or unofficial, supported by professionals in the field, in Spanish, and at all times related to the subject in question, all with the primary intention that such information serves to provide fundamental knowledge to the interested party, and in particular for this investigation, on the basis of the conclusions that will be developed next, and formative contribution for possible investigations of the subject.
\end{abstract}

Keywords: Technologies, information, communication, networks, models. 


\section{Redes Informáticas aplicadas a la Educación}

Vol. 2, núm. 2., (2018)

Gladys Patricia Guevara Albán; Cristian Salomón Guevara Albán; Alexis Eduardo Verdesoto

Arguello

\section{Introducción.}

Siguiendo a Chiscueth (2016) se puede iniciar diciendo que en el mundo actual la sociedad transforma su forma de vivir según sus necesidades, en tal sentido, hace uso de lo que hay a su disposición, y la educación es una base de partida para la transformación humana.

Hasta hace algunos años las tecnologías de la información se han adentrado poco a poco en el uso de la enseñanza de diferentes áreas.

El uso de herramientas tecnológicas en la educación ha ayudado a que los estudiantes aprendan de una forma diferente a la tradicional. Aun así, los medios convencionales como la pizarra, el retroproyector, los rotafolios siguen utilizándose, los medios audiovisuales y tecnológicos se consideran también motivacionales para el logro del aprendizaje de los alumnos en cualquier nivel de educación, asegura Sánchez (s.f.)

La misma autora agrega que el hecho de trabajar con las computadoras y el hacer uso del acceso a Internet para aprender y enseñar, ha creado un impacto sorprendente, sobre todo porque actualmente la mayoría de las actividades giran en torno al uso de la tecnología. Existen diferentes medios tecnológicos para la enseñanza y el aprendizaje entre los que se pueden mencionarlas plataformas educativas que son entornos virtuales que se apoyan de sistemas informáticos y en donde el Internet es requisito primordial.

En razón de lo expuesto es que se puede asegurar que las TIC's han venido a ampliar la oferta educativa, en razón de que es posible ofrecer nuevos modelos de enseñanza-aprendizaje, desde el modelo presencial hasta el modelo a distancia, sin dejar de mencionar la modalidad 


\section{Redes Informáticas aplicadas a la Educación}

Vol. 2, núm. 2., (2018)

Gladys Patricia Guevara Albán; Cristian Salomón Guevara Albán; Alexis Eduardo Verdesoto Arguello

mixta en donde los participantes realizan parte de sus actividades en el aula y otras actividades apoyándose del Internet y es aquí donde entra el uso de las plataformas educativas.

Aunado a lo anterior, Salinas (1999) manifiesta que las redes informáticas se han ido convirtiendo en un fenómeno social mediante un imparable proceso de comercialización. Después de haber estado en manos de una élite de científicos e intelectuales, el ciberespacio vuelve a estar dominado por grandes empresas de la comunicación audiovisual y del ocio que se erigen en emisores privilegiados, concentrando el control sobre los contenidos y sobre las audiencias (segmentadas, individualizadas...). Pero junto a esta organización, convive la comunicación horizontal, el intercambio, los emisores-receptores que vienen siguiendo la tradición de lo que a través de redes se ha venido haciendo. En la medida que puedan convivir ambos aspectos, la red tendrá un gran potencial educativo, porque eso permitirá un gran flujo de comunicación institucional, personal, informal.

El autor agrega que, dadas las posibilidades de dicho fenómeno, recaba su atención, ya que la comunidad educativa con sus modos, medios y técnicas necesita adaptarse a una sociedad cada vez más apoyada en las TIC's y desde esta perspectiva el fenómeno de las redes debe ser analizado, investigado y experimentado para la enseñanza. Asegura que, es desde esa perspectiva desde la que se piensa sobre las redes en la educación, sobre las posibilidades de crecimiento de la intercomunicación, la comunicación horizontal, multidireccional que ha caracterizado a Internet. 


\section{Redes Informáticas aplicadas a la Educación}

Vol. 2, núm. 2., (2018)

Gladys Patricia Guevara Albán; Cristian Salomón Guevara Albán; Alexis Eduardo Verdesoto

Arguello

En cualquier caso, lo que sí podemos dar por seguro es que la educación parece ser uno de los campos privilegiados de explotación de las posibilidades comunicativas de las redes informáticas, y ante semejantes perspectivas los pedagogos no podemos quedar indiferentes.

\section{Materiales y métodos.}

\section{Materiales}

Materiales de escritorio, material bibliográfico, materiales audiovisuales, materiales para impresión.

\section{Métodos}

En vista de que lo que se pretende con este trabajo es describir, definir términos y conceptualizar en líneas general respecto al uso de las tecnologías de la información y comunicación (TIC's) en la comunidad educativa, haciendo énfasis en la utilización de las redes informáticas en las aulas de clase, vistas como una herramienta pedagógica hoy en día indispensable para el fortalecimiento de las actividades curriculares, se ha propuesto el desarrollo de una investigación de tipo documental, a nivel exploratoria y de corte longitudinal.

Respecto a la investigación Documental, (Baena, 1985) citado en Ávila (2006), la define como "una técnica que consiste en la selección y recopilación de información por medio de la lectura y crítica de documentos y materiales bibliográficos, de bibliotecas, hemerotecas, centros de documentación e información" (p. 72). 


\section{Redes Informáticas aplicadas a la Educación}

Vol. 2, núm. 2., (2018)

Gladys Patricia Guevara Albán; Cristian Salomón Guevara Albán; Alexis Eduardo Verdesoto Arguello

Franklin (1997), igualmente citado en Ávila (2006), respecto a la investigación documental aplicada a la organización de empresas como una técnica de investigación, dice que "se deben seleccionar y analizar aquellos escritos que contienen datos de interés relacionados con el estudio" (p. 13).

Según Ávila (2006) en los Estudios Longitudinales la unidad de análisis es observada en varios puntos en el tiempo.

Parafraseando a Ríos (2017) se entiende que la investigación se cataloga como documental porque responde a una fuente de información, puesto que se apela a documentos para alcanzar los resultados planeados; y en cuanto al nivel de conocimiento, la investigación es descriptiva, cuando busca encontrar las características, comportamiento y propiedades del objeto de estudio en el presente, no ha futuro, siendo en ese último caso que se denomina investigación de pronóstico.

\section{Resultados.}

\section{Uso de las TIC's en Educación}

Algunos de los ejemplos del uso de las TIC en educación son la pizarra digital, las tablets, las mesas interactivas, los libros digitales, las aplicaciones educativas y las plataformas de enseñanza digital.

La incorporación de las nuevas tecnologías a nuestra vida ha cambiado el mundo en el que vivimos por completo. Entre esas modificaciones encontramos las que se han producido en 


\section{Redes Informáticas aplicadas a la Educación}

Vol. 2, núm. 2., (2018)

Gladys Patricia Guevara Albán; Cristian Salomón Guevara Albán; Alexis Eduardo Verdesoto

Arguello

el ámbito educativo, en el cual en la actualidad, es extraño ver una clase en la que no se utilicen para mejorar el proceso de enseñanza-aprendizaje.

Tapscott (1997) ofrece algunos ejemplos del uso de las TIC’s en la educación, entre las cuales menciona:

Pizarra digital interactiva: es una herramienta formada por un ordenador, un proyector y una pantalla que permite ser controlada por un puntero. Existen diferentes tipos de pizarra, aunque la más utilizada es la que se acaba de describir, está surgiendo otra, que es la pizarra digital interactiva táctil. Se diferencia de la anterior en que nos permite controlar la pantalla con nuestros dedos. La pizarra digital nos permite realizar muchísimas actividades, por ejemplo; se puede proyectar cualquier información que provenga del portátil ya que la pizarra se convierte en un gran monitor en el que podemos oír y ver de forma ampliada los contenidos de éste. Entre esos contenidos los más comunes suelen ser: programas generales y educativos, vídeos, música, páginas web, presentaciones, documentos, etc. Además, se puede interactuar con ellos de la misma manera que lo haríamos con el ordenador sin pizarra digital. También podemos proyectar en ella contenidos de las herramientas multimedia que presentaremos más adelante si lo conectamos adecuadamente mediante un cable: CD, DVD, cámara de fotos. Las pizarras incorporan sus propias herramientas y recursos didácticos que van destinados a todas las edades y áreas. Por otro lado, también podemos utilizar los bancos de imágenes, sonidos y música o si lo preferimos utilizar los portales y webs que existen con el fin de cumplir nuestros objetivos.

Mesas interactivas: aunque está menos extendida esta herramienta, se puede utilizar con grupos de niños en la etapa infantil. Una de sus ventajas es que dado su diámetro de 27pulgadas 


\section{Redes Informáticas aplicadas a la Educación}

Vol. 2, núm. 2., (2018)

Gladys Patricia Guevara Albán; Cristian Salomón Guevara Albán; Alexis Eduardo Verdesoto Arguello

puede ser utilizado hasta los seis niños, los cuales trabajarán con contenido digital como si de una pizarra digital se tratara. Ésta ha sido diseñada para que los alumnos tengan un momento de reflexión y debate entre ellos gracias al contenido digital que van trabajando. Les suele gustar mucho porque pueden tocar la pantalla y todos los juegos que incorpora son interactivos. Sus funciones son las mismas que las de cualquier otra herramienta digital. La diferencia se encuentra en que para facilitar su uso su altura está adaptada a la de los más pequeños. Por otro lado, también incorpora juegos educativos con los que los niños podrán aprender mientras se divierten de forma intuitiva y utilizando los dedos. Sin embargo, debido a su alto precio y a que sólo tiene aforo para seis personas, no está muy generalizada.

Tableta: son pequeños dispositivos que se caracteriza por tener un tamaño intermedio entre el portátil y el teléfono móvil. Algunos centros los han incorporado para trabajar con él en algunas asignaturas debido a su diseño, más ligero e intuitivo que los portátiles. Por otro lado, tampoco necesitan complementos ni accesorios lo que a diferencia del portátil hace que los centros se ahorren dinero. Las funciones de esta herramienta son las mismas que las de cualquier dispositivo. La diferencia está en la interactividad mediante la pantalla, que se puede manejar con un lápiz electromagnético. Para aquellos alumnos que tengan alguna discapacidad visual, puede ser una herramienta muy útil, ya que puede trabajar sobre ella pudiendo adaptar de esta forma las letras y el entorno del dispositivo a sus necesidades e incluso si lo requiere utilizar los programas que existen para este tipo de discapacidad. Para los alumnos de los últimos cursos de Educación Infantil y los primeros de Educación Primaria es muy útil la función de la escritura sobre la pantalla, porque les permite el aprendizaje de la escritura en un medio tan motivador como es el mundo digital. 


\section{Redes Informáticas aplicadas a la Educación}

Vol. 2, núm. 2., (2018)

Gladys Patricia Guevara Albán; Cristian Salomón Guevara Albán; Alexis Eduardo Verdesoto

Arguello

Libros digitales: son herramientas con contenido de tipo textual digital para las que se debe de utilizar una pantalla. Normalmente su uso en el aula va acompañado de otro tipo de contenidos como es el audiovisual o incluso el online. Su incorporación en el aula ha traído consigo mucha polémica en el entorno educativo, ya que algunas personas pensaban que su uso dentro del aula iba a sustituir al papel en todos los sentidos y que por ende, iba a perjudicar a los niños en el aprendizaje de la lectura y la escritura. Sin embargo, al igual que otras herramientas ofrecen grandes beneficios en el proceso de enseñanza-aprendizaje.

Portátiles: se podría decir que fueron las primeras herramientas que llegaron al aula después del cañón, la televisión, el vídeo... Al igual que las demás herramientas, permite el trabajo tanto individual como en grupo y motiva a los estudiantes de forma excelente en las actividades que realizan en él. Por otro lado, es un buen complemento de aprendizaje para que los niños interioricen lo que han aprendido sobre cualquier tema en concreto o incluso para ampliarlo si así lo prefieren. Por ser un instrumento lúdico, los niños no saben distinguir si están jugando o trabajando con el ordenador, pero jueguen o trabajen, lo que sí es cierto es que aprenden a utilizarlo y adquieren nuevos conocimientos, de modo que en las escuelas se dispone cada vez de más programas y aplicaciones pedagógicas de alta calidad para ofrecerles.

Software específico de aplicaciones online o actividades online: con la llegada de las nuevas tecnologías también nos ha sido posible utilizar diferentes programas, plataformas educativas o páginas en las que existen actividades de tipo online que podemos utilizar para trabajar las diferentes áreas de conocimiento. De esta forma podemos hacer que el aprendizaje de aquellas asignaturas que puedan resultar más aburridas o difíciles de entender se conviertan en algo muy divertido. Aunque siempre se recomienda que este tipo de actividades se utilicen una 


\section{Redes Informáticas aplicadas a la Educación}

Vol. 2, núm. 2., (2018)

Gladys Patricia Guevara Albán; Cristian Salomón Guevara Albán; Alexis Eduardo Verdesoto Arguello

vez que se les ha explicado el contenido principal, algunos profesores ya las están incorporando también en el momento de la explicación para captar la atención de los discentes. Por otro lado, también se pueden utilizar como apoyo a los deberes que se les manda a los niños en formato papel. En muchas ocasiones, nuestros alumnos adquirirán mejor el aprendizaje si manejan algún dispositivo para realizar este tipo de actividades por ser más interactivo y dinámico el procedimiento.

Los multimedia: los recursos multimedia son aquellas herramientas que siempre se han utilizado en el aula para hacer menos monótono el aprendizaje. Es decir, las que hemos utilizado desde siempre antes de que llegaran aquellas más modernas como las que hemos estado mencionando anteriormente. Algunos ejemplos son: la televisión, el vídeo, DVD, el cañón... Actualmente, éstas se utilizan como complemento a las otras herramientas más modernas, sin embargo, he creído conveniente mencionarlas porque han sido la base para el desarrollo de todas las demás.

Plataformas de enseñanza virtual: otra herramienta bastante conocida en el campo educativo por la cantidad de beneficios que brinda a los estudiantes son las plataformas de enseñanza virtual, entendida como aquella herramienta que permite al alumno estudiar la materia a distancia sin la necesidad de desplazarse al centro de formación. Esto ha permitido diferentes modalidades de estudio como el e-learning o aprendizaje electrónico en español o el b-learning o aprendizaje semipresencial.

Respecto al uso de las TIC's en Educación, Sánchez (2018)describe algunas ventajas y desventajas: 


\section{Redes Informáticas aplicadas a la Educación}

Vol. 2, núm. 2., (2018)

Gladys Patricia Guevara Albán; Cristian Salomón Guevara Albán; Alexis Eduardo Verdesoto

Arguello

\section{Ventajas}

- Su uso en el aula incrementa la motivación de los alumnos. Es un hecho que desde que se comenzaron a utilizar en las aulas los alumnos se encuentran más motivados en las diferentes áreas que estudian.

- $\quad$ Renueva los métodos de aprendizaje y los procesos. En la actualidad, todos los profesionales de la educación han tenido que reciclarse para utilizar estas herramientas en las aulas y cambiar la forma en la que enseñan.

- $\quad$ Permite utilizar nuevos recursos educativos. Gracias a la continua evolución de las nuevas tecnologías poco a poco se van incorporando en las aulas nuevos dispositivos que aumentan la calidad de la enseñanza.

- Se aprovecha más el tiempo en clase. Con la multitud de actividades online que existen, los alumnos pueden aprender más contenido en menos tiempo.

- Implica al alumno en las tareas. Los alumnos aprenden jugando y sin darse cuenta los contenidos que se trabajan en clase.

- Fomentan la colaboración entre los alumnos. Algunas herramientas como las mesas táctiles permite que los discentes colaboren en la resolución de problemas.

- Alfabetización digital y audiovisual. Permiten a los alumnos adquirir las competencia digitales y audiovisuales necesarias para su futuro.

- Otra de las ventajas son: Estimula la creatividad, respeta el ritmo de aprendizaje de los alumnos, crean curiosidad y espíritu de investigación, pueden experimentar y manipularlos.

\section{Desventajas}




\section{Redes Informáticas aplicadas a la Educación}

Vol. 2, núm. 2., (2018)

Gladys Patricia Guevara Albán; Cristian Salomón Guevara Albán; Alexis Eduardo Verdesoto Arguello

- El precio de algunas de estas herramientas es muy elevado e impiden su generalización.

- $\quad$ Algunos sólo pueden colocarse de una determinada forma dentro del aula como es el caso de la pizarra digital.

- $\quad$ Por las características del aula puede existir falta de luminosidad si hay luz en el aula o si el proyector no es suficiente potente.

- También se pueden proyectar las sombras sobre la pizarra por parte de alumnos y profesores.

- $\quad$ Pueden existir problemas técnicos con el acceso a internet, los ordenadores o el software de la pizarra digital, por ejemplo.

- Supone una inversión de tiempo inicial mayor en la preparación de las actividades en los docentes.

- Se necesita cierto grado de conocimiento para la elaboración de actividades propias, por lo que si el profesorado no está reciclado y no sabe manejarlas adecuadamente puede ser un gran problema.

- Puede existir un exceso de información para los alumnos en el uso de estos dispositivos.

- En algunas ocasiones puede distraer a los alumnos si no se sabe llevar la clase mientras se usa este tipo de tecnología.

La introducción de las TIC en el sector educativo viene enmarcada por una situación de cambios: cambios en los usuarios de la formación, cambios en los entornos o escenarios de aprendizaje, cambios en los modelos y concepciones. Las circunstancias tecnológicas, culturales 


\section{Redes Informáticas aplicadas a la Educación}

Vol. 2, núm. 2., (2018)

Gladys Patricia Guevara Albán; Cristian Salomón Guevara Albán; Alexis Eduardo Verdesoto

Arguello

y sociales en las que se desenvuelve la actual sociedad exigen, ya, cambios en todos los elementos del proceso didáctico, incluidos nuevos objetivos para la educación.

El influjo de la evolución de la sociedad, a la que ha contribuido sin duda la evolución misma de las tecnologías de la información, requiere plantear estos nuevos objetivos. En el futuro la obtención y organización de la información se convertirá en la actividad vital dominante para mucha gente. Al mismo tiempo que contribuyen al vertiginoso cambio que exige nuevas destrezas y cambios en los objetivos, deben contribuir a su logro. Ello constituye uno de sus grandes desafíos, atender a las nuevas necesidades educativas que la evolución de la sociedad y la evolución misma de las TIC generan, y la anticipación de las necesidades educativas que la evolución futura planteará.

En este contexto, surgen diversos elementos de reflexión relacionados con el impacto de las TIC en la educación y los cambios que en esa se generan para adaptarse, entre los que podemos destacar:

\section{Cambios en el ciudadano}

Las posibilidades que las redes ofrecen en la formación hacen que surjan nuevos usuarios-alumnos caracterizados por una nueva relación con el saber y nuevas prácticas de aprendizaje adaptables a situaciones cambiantes.

Lograr este tipo de personas flexibles para adaptarse a situaciones de continuo cambio, responder a los desafíos que la evolución de la tecnología, la cultura y la sociedad plantea, va a depender de la puesta en marcha de acciones educativas relacionadas con el uso, selección, 


\section{Redes Informáticas aplicadas a la Educación}

Vol. 2, núm. 2., (2018)

Gladys Patricia Guevara Albán; Cristian Salomón Guevara Albán; Alexis Eduardo Verdesoto Arguello

utilización y organización de la información de manera que el alumno vaya formándose como un maduro ciudadano de la sociedad de la información.

No se trata de convertir a los ciudadanos en especialistas en comunicaciones, sino que la cultura de la comunicación esté en la formación básica para que la persona pueda desarrollar mejor sus posibilidades individuales y profesionales. Los cambios generan dificultades e incertidumbres, pero también nuevos espacios de oportunidades. Por eso hay que adaptarse y crear esos nuevos espacios.

Paralelamente, se hace necesaria una formación del profesorado en la misma dirección. Esto es: dominio de las tecnologías (no para enseñarlas, sino como usuario aventajado) y preparación para la función de guía y orientación en el uso y consumo de la información.

En este contexto podemos considerar que una persona educada debe ser un conocedor, un pensador y un aprendiz. En otras palabras, ser (o estar) educado supone la adquisición de un dominio específico de conocimiento, la posesión de destrezas cognitivas generalmente útiles y la habilidad y deseo de aprender.

\section{Cambios en los escenarios del aprendizaje}

Al analizar los posibles escenarios propiciados por las redes informáticas tendremos que distinguir entre los cambios que puedan producirse en el ámbito de la enseñanza convencional de aquellos escenarios que se ven fuertemente potenciados por el uso educativo de las redes y que caen preferentemente en el ámbito de la enseñanza flexible y a distancia. En general y con la actual tecnología, al menos en un futuro próximo, no parece que vayan a incidir en la enseñanza 


\section{Redes Informáticas aplicadas a la Educación}

Vol. 2, núm. 2., (2018)

Gladys Patricia Guevara Albán; Cristian Salomón Guevara Albán; Alexis Eduardo Verdesoto

Arguello

básica y media. Incidir, en el sentido de transformar el sistema. Se irá introduciendo como un recurso más, como un importante banco de recursos tal como hemos visto, sin provocar cambios importantes en la forma de enseñar. Constituyendo, eso sí, un preciado recurso para profesores y alumnos. Quizá esta forma constituya la aportación más positiva de las redes a la educación básica.

Junto a la explotación como fuente de recursos dentro de la institución educativa, debemos atender al efecto que pueda tener el cada vez más creciente acceso doméstico en fórmulas que pueden caer en el ámbito de la educación informal. Es indudable que los alumnos que utilizan Internet, en cualquiera de ambas fórmulas, se benefician de varias maneras: mejoran su contacto con la informática y la tecnología; aprenden a trabajar en un mundo transnacional; se desenvuelven en otros idiomas; tienen acceso a miles de informaciones antes inalcanzables (bancos de datos, catálogos, museos, bolsas de trabajo internacionales, etcétera).

\section{Cambios en los modelos}

Las posibilidades de las redes para la enseñanza superior en el contexto de estos nuevos escenarios (formación encaminada a mejorar la competencia profesional de manera constante, renovación, ampliación y puesta al día del conocimiento científico y de las habilidades técnicas necesarias para mantener los estándares profesionales lo más alto posible, la formación de los profesores en procesos de tipo colaborativo), todavía no han sido explotadas.

Es indudable la asistencia a una inflación, tanto de cursos on-line, como de aprendizaje abierto. Cualquier persona con una conexión a Internet puede apuntarse a los cientos de cursos 


\section{Redes Informáticas aplicadas a la Educación}

Vol. 2, núm. 2., (2018)

Gladys Patricia Guevara Albán; Cristian Salomón Guevara Albán; Alexis Eduardo Verdesoto Arguello

de idiomas, mecanografía o informática que aparecen en la red. También en el terreno de la formación continua y superior se observa un incremento de las demandas de aprendizaje.

Este incremento del mercado de formación lleva consigo mayores oportunidades y mayor competitividad, tanto para las instituciones existentes, como para las de nueva creación.

Hanna (1998), en (Salinas, 1999) dice que se pueden diferenciar distintos modelos de enseñanza apoyados en las posibilidades que hoy brindan las redes para la formación, entre las cuales están:

a. Universidades de educación a distancia basadas en la tecnología. Se trata de las universidades on-line o universidades virtuales cuyo número está creciendo constantemente y que ofrecen un modelo de enseñanza organizado en función de la tecnología (utilizando fundamentalmente sistemas de aprendizaje asíncrono, apoyándose en las ventajas de los sistemas de conferencia mediante ordenador y en las posibilidades crecientes de world-wide-web).

b. Instituciones privadas dirigidas a adultos. La formación de adultos es uno de los mercados emergentes para universidades privadas y organizaciones o empresas privadas existentes y nuevas que operan dirigiéndose a segmentos muy determinados de ese mercado, atentos a las necesidades que presentan, en ámbitos como el de la cualificación técnica o de gestión, ofreciendo cursos estándar, etc.

c. Universidades corporativas. Se trata de organizaciones de formación que diversas corporaciones empresariales han puesto en funcionamiento para cubrir necesidades de formación específica de sus propios recursos humanos (desarrollo de competencias base 


\section{Redes Informáticas aplicadas a la Educación}

Vol. 2, núm. 2., (2018)

Gladys Patricia Guevara Albán; Cristian Salomón Guevara Albán; Alexis Eduardo Verdesoto

Arguello

para el puesto de trabajo; integración en la cultura de la compañía; aumento de la cooperación, la comunicación y las competencias de los empleados de forma individual y en equipo, etc.) y que proporcionan estudios que tienen acreditación.

d. Alianzas estratégicas universidad-industria. La asociación y las alianzas estratégicas se están desarrollando entre universidades y organizaciones con ánimo de lucro. Esto supone el contacto entre diferentes culturas organizativas, diferentes objetivos, y diferentes principios operativos.

e. Organizaciones de control de acreditación y certificación. La emergencia de un extenso mercado de formación continua ha hecho surgir organizaciones que se encargan de certificar las competencias individuales (adquiridas autónomamente o a través de programas de formación), tanto de cara a los trabajadores, como a los empresarios.

f. Universidades tradicionales extendidas. Muchas universidades convencionales diseñan y organizan programas específicos para ofrecerlos a una audiencia de adultos en modalidades no presenciales. Las experiencias van desde unidades poco dependientes de la organización tradicional y que deben autofinanciarse, hasta universidades que modifican y ofrecen los programas convencionales para una audiencia no convencional. Se trata, en cualquier caso, de atender a un mercado de formación continua que cada vez requiere mayor especialización.

g. Universidades multinacionales globales. Generalmente apoyados en alguna universidad convencional (universidad tradicional extendida o universidad a distancia basada en la tecnología), se trata de servicios de formación superior de carácter internacional $-\mathrm{o}$ mejor global — que se pueden apoyar o no en universidades de los otros países. 


\section{Redes Informáticas aplicadas a la Educación}

Vol. 2, núm. 2., (2018)

Gladys Patricia Guevara Albán; Cristian Salomón Guevara Albán; Alexis Eduardo Verdesoto Arguello

El éxito de cualquiera de estos tipos de proyectos dependerá de varios factores (Salinas, 1997): el prestigio de las instituciones, la flexibilidad del profesorado (su capacidad para adaptarse a las condiciones impuestas por las nuevas tecnologías), calidad de los contenidos frente a los fuegos de artificio multimedia, interactividad no limitada a profesor-alumno, reconstrucción de los ambientes de comunicación humana.

La importancia en la educación de las redes de computadoras proviene de su capacidad para usar las redes posibilidades de comunicación. Nuevas experiencias y proyectos aparecen donde los estudiantes se están convirtiendo en grandes usuarios de estas redes. Los educadores no pueden permanecer neutrales a estos cambios. El impacto de las tecnologías de la información y la comunicación en la educación no está limitado a su efecto cuando se usan en experiencias de enseñanza-aprendizaje. Una hoja de ruta más amplia para el se requiere un cambio que involucre a los usuarios, el entorno de aprendizaje, los modelos educativos, etc. Los cambios en tecnología y cultura en la sociedad actual requieren cambios en los modelos educativos.

\section{Conclusiones.}

Existen muchas herramientas TIC's que se pueden utilizar en el aula para motivar a los alumnos y mejorar su aprendizaje. Sin embargo, no porque las utilicemos todas seremos buenos profesionales y éstos aprenderán más contenidos. Tenemos que ser capaces de saber elegir qué herramientas vamos a utilizar y para qué y con qué fin, ya que todas no serán igual de eficaces y efectivas para la comprensión de un determinado contenido por parte de nuestros discentes.

Merchán (2017) asegura que el uso de las TIC's se ha vuelto esenciales para el desarrollo en varios campos, pues todos estos son utilizados a diario para realizar diversas actividades en 


\section{Redes Informáticas aplicadas a la Educación}

Vol. 2, núm. 2., (2018)

Gladys Patricia Guevara Albán; Cristian Salomón Guevara Albán; Alexis Eduardo Verdesoto

Arguello

varios lugares e instituciones del Ecuador. En nuestro país comienza a ganar experiencia en ésta área, puesto que en el campo educativo se viene fortaleciendo tecnológicamente, por mencionar un ejemplo, la creación de las Unidades Educativas del Milenio, es un proyecto muy atractivas, pues se promueve la institución educativa modelo, que debería tener todo tipo de condiciones idóneas para quienes estudian en ellas, es decir los suficientes laboratorios de computación, física, química y que disponga de conexión a internet, pizarras didácticas en todas las aulas, por lo que entonces, la implementación de estas escuelas sin duda alguna contribuyen al desarrollo del país siempre que se cumpla el objetivo de las mismas, los cuales son educar para el futuro de la patria, donde los jóvenes se desarrollen en diversas áreas de la mano de la tecnología, la investigación e innovación.

Gracias a avances como este se han obtenido ciertas ventajas en el ámbito educativo, una de ellas es que estos proyectos resultan atractivos para el desarrollo de su formación académica, debido no sólo a la tecnología material sino principalmente al acceso de información en los sitios web a nivel mundial, lo cual ayuda en las múltiples tareas a desarrollar según el plan de estudios, pues si bien es positivo que los estudiantes de esta época puedan tener mayor accesibilidad la información, no es menos importante los procesos de aprendizaje que se desprenden de la información obtenida por estos medios, lo que significa que no se deja de un lado el análisis, más bien, debe permanecer la interacción entre la información a la que se accede y el empleo de las herramientas tradicionales de aprendizaje. Lo significativo ahora es que, el proceso apredizajeenseñanza se vuelven más dinámico y llamativo puesto que nace una mayor motivación para el trabajo académico y la creatividad al ejecutarlo, ya que los estudiantes tratan de sobresalir ingeniando sus tareas, esto hace que se vuelvan actores y dramaticen si se trata de exponer algún 


\section{Redes Informáticas aplicadas a la Educación}

Vol. 2, núm. 2., (2018)

Gladys Patricia Guevara Albán; Cristian Salomón Guevara Albán; Alexis Eduardo Verdesoto Arguello

tema de historia y los plasmen en un video, o hagan una grabación simulando hacer un programa radial, en fin, depende mucho de la creatividad y del apoyo docente, ya que la pedagogía acertada definirá también mucho en su formación.

\section{Recomendaciones.}

Se cree relevante que en un futuro se investigue en esta misma línea, sobre los resultados obtenidos a corto plazo por la utilización de las redes informáticas en las aulas de clase en los distintos niveles educativos, aplicadas una herramienta pedagógica o andragógica, según el caso, para el fortalecimiento de las actividades curriculares.

\section{Bibliografía.}

Ávila, H. (2006). Introducción a la Metodología de la Investigación. eumed.net.

Chiscueth, P. (Octubre de 2016). TIC'S en la educación. Educación Básica. (UTC, Ed.) Obtenido de http://repositorio.utc.edu.ec/handle/27000/3830

Merchán, E. (2017). Uso de las tics, plataformas digitales y medios interactivos en las unidades educativas del milenio: un estudio de caso. Obtenido de http://repositorio.utmachala.edu.ec/handle/48000/10613

Ríos, R. (2017). Metodología para la Investigación y Redacción (Primera ed.). Malaga: Servicios Académicos Intercontinentales S.L.

Salinas, J. (1999). Uso educativo de las redes informáticas. EDUCAR, 25, 81-92.

Sánchez, C. (s.f.). Educación y TIC's. Bogotá.

Sánchez, Y. (2018). www.lifeder.com. Obtenido de https://www.lifeder.com/ejemplos-ticseducacion/

Tapscott, D. (1997). Creciendo en un entorno digital. Bogotá: Mc Graw-Hill. 